\title{
RISK-BASED \\ SPACECRAFT FIRE SAFETY EXPERIMENTS 3
}

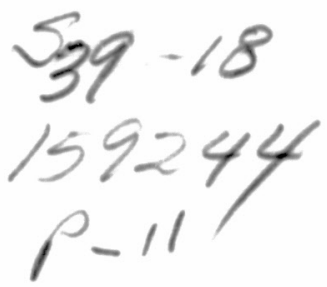

\author{
G. Apostolakis, I. Catton, F. Issacci, \\ T. Paulos, S. Jones, K. Paxton and M. Paul
}

Mechanical, Aerospace and Nuclear Engineering Department University of California, Los Angeles

Flight Experiments Technical Interchange Meeting Monterey, California, October 5-9, 1992

Sponsored by NASA Lewis Research Center 


\section{PROBABILISTIC RISK ASSESSMENT APPLIED TO FIRE SAFETY}

Spacecraft fire risk can never be reduced to a zero probability.

Probabilistic risk assessment is a tool to reduce risk to an acceptable level.

\section{MAJOR STEPS:}

1. Identification of "critical" locations and the assessment of the frequency of fires: overheating, spills, smoldering, ignition, etc.

2. Estimation of the fraction of fires that can lead to damage of specified components: fire growth time and the competing detection and suppression times

3. Estimation of the fraction of fires that can lead to mission damage 


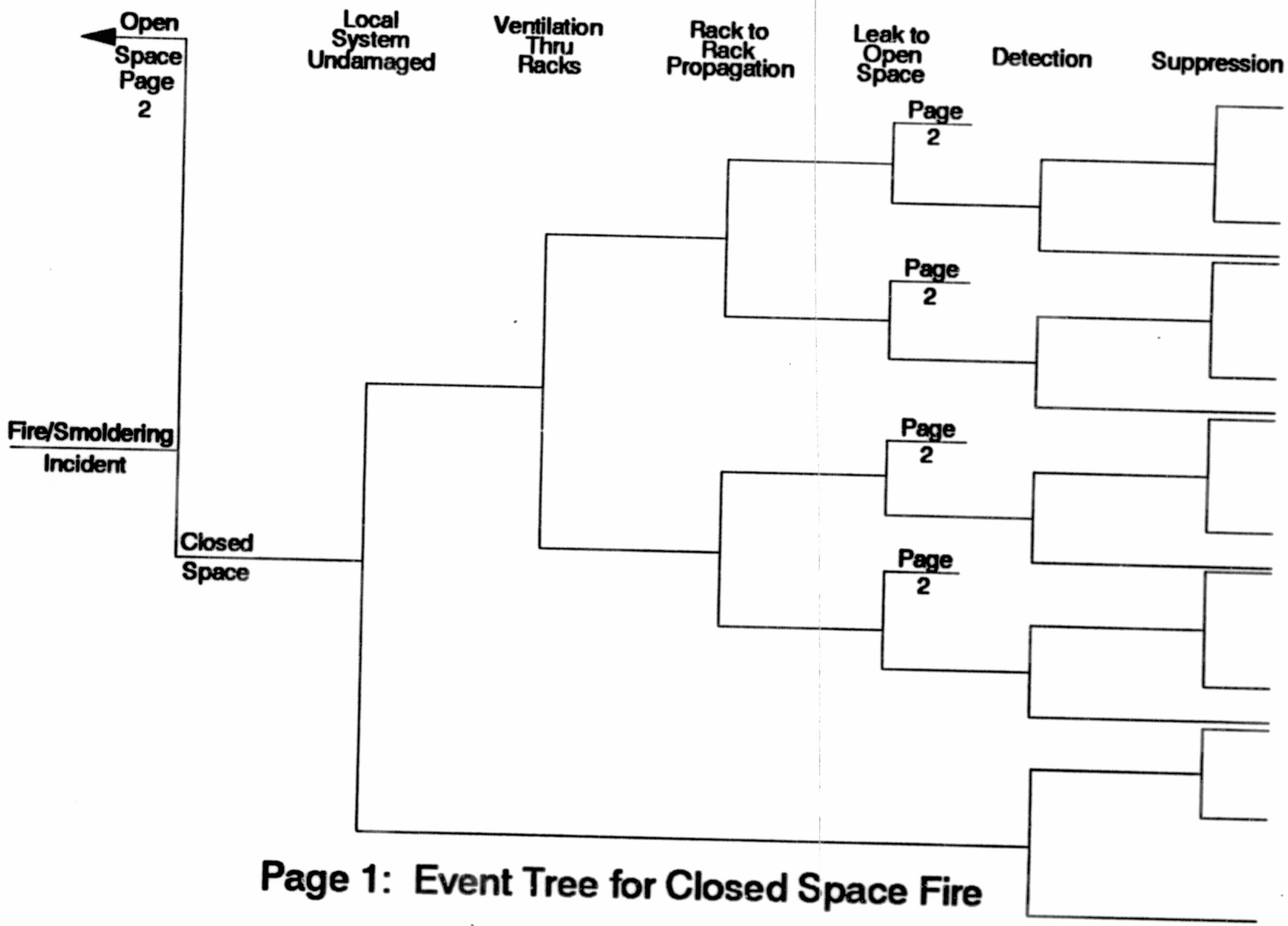




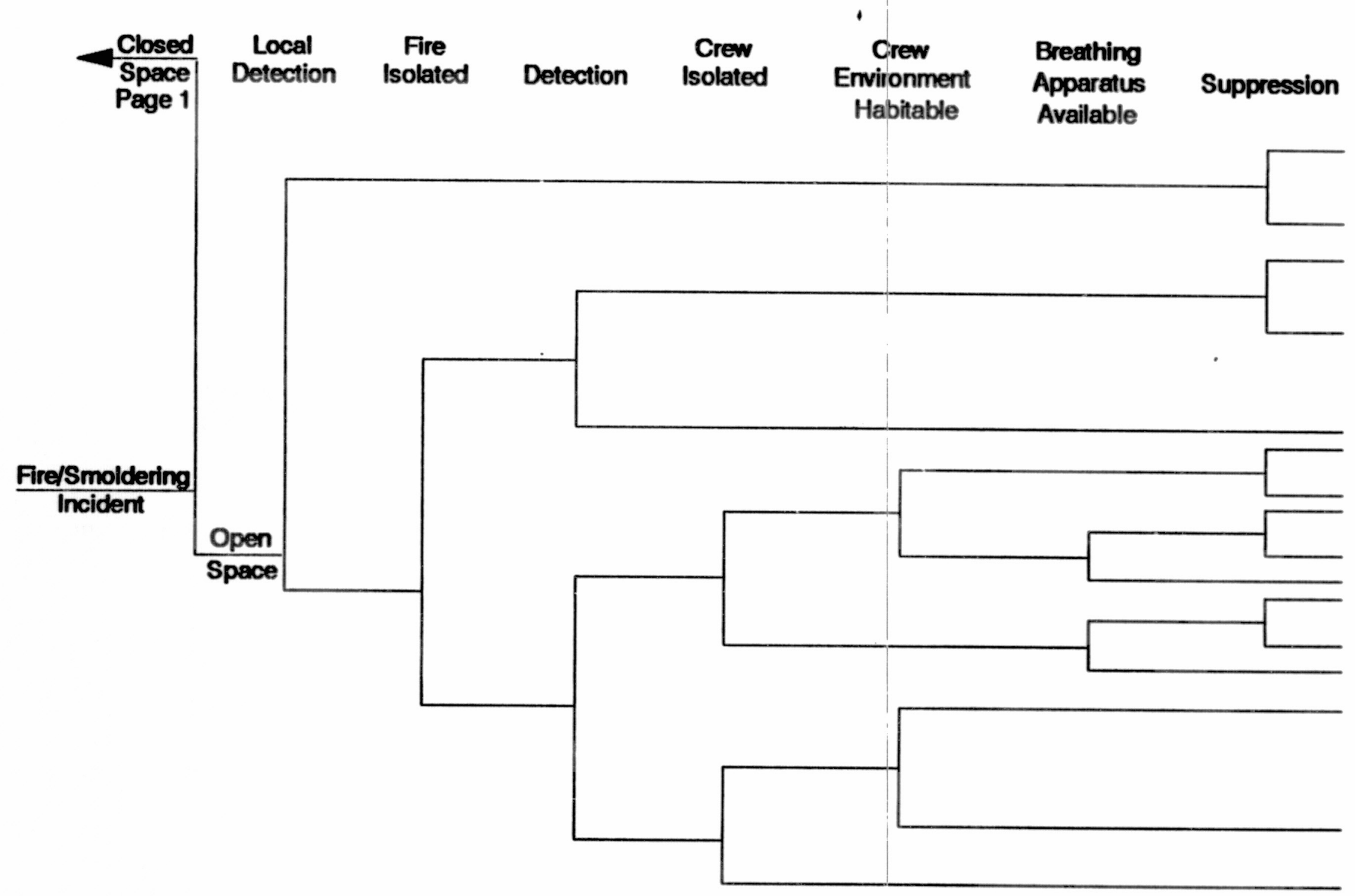

Page 2: Event Tree for Open Space Fire 


$$
\lambda_{\text {loss }}=\sum \lambda_{\mathrm{j}} \mathrm{Q}_{\mathrm{d} / \mathrm{j}, \mathrm{k}} \mathrm{Q}_{\text {loss } \mathrm{d} / \mathrm{j}, \mathrm{k}}
$$

$\lambda_{\text {loss }} \quad$ frequency lost

$\lambda_{j} \quad$ frequency of class $j$ fires

$\mathrm{Q}_{\mathrm{d} / \mathrm{j}, \mathrm{k}} \quad$ fraction of class $\mathrm{j}$ fires that lead to damage of the $\mathrm{k}^{\text {th }}$ critical system

$Q_{\text {loss } d / j, k}$ fraction of class $\mathbf{j}$ fires leading to damage of the $\mathbf{k}^{\text {th }}$ system that cause the loss of the spacecraft

$$
\mathrm{Q}_{\mathrm{d} / \mathrm{j}}=\operatorname{Fr}\left[\mathrm{T}_{\mathrm{G}}<\mathrm{T}_{\mathrm{H}} / \text { fire }\right]
$$

$T_{G} \quad$ growth time

$\mathrm{T}_{\mathrm{H}} \quad$ hazard time

$$
T_{H}=T_{f}+T_{d}+T_{s}
$$

$\mathrm{T}_{\mathrm{f}} \quad$ time to detection

$T_{d} \quad$ detector response time

$\mathrm{T}_{\mathrm{s}} \quad$ suppression time 


\section{Source - Transport - Deposition}

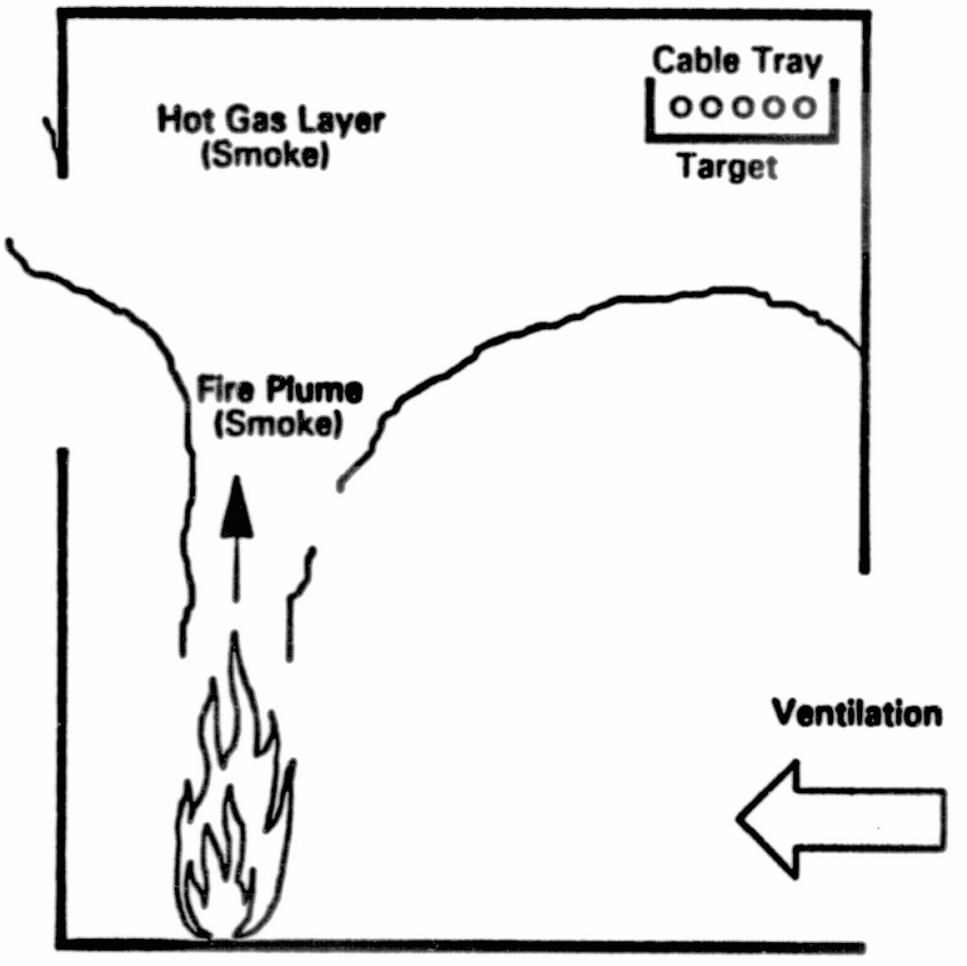

Terrestrial

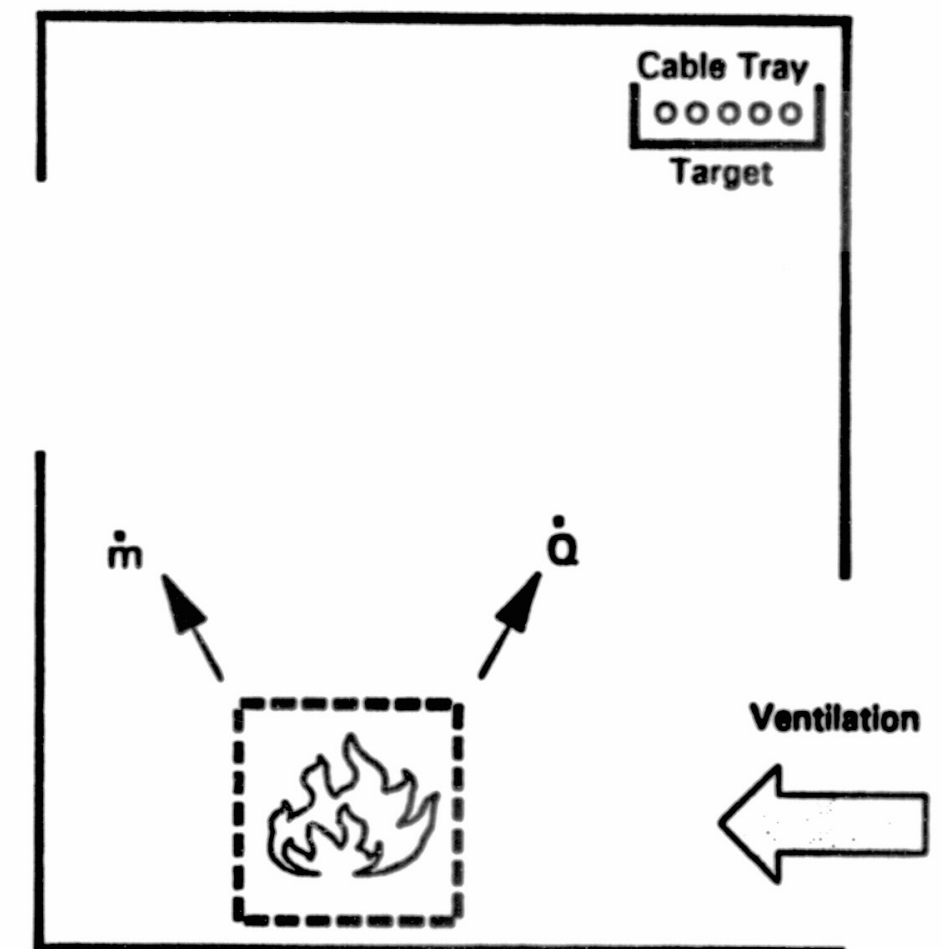

Microgravity 


\section{Fire Safety Assessment}

Target Identification
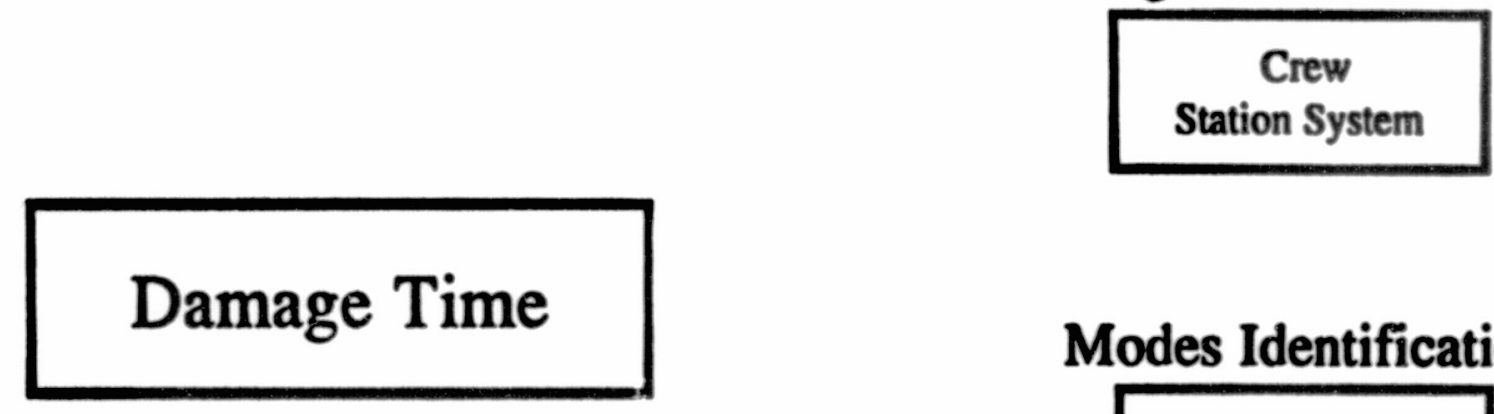

Detection \& Suppression Time

Modes Identification

\begin{tabular}{|c|}
\hline Heat \\
Smoke \\
Toxins \\
\hline
\end{tabular}

Event Description

Source

Transport

Deposition 


\section{Wire Overload Phenomena}

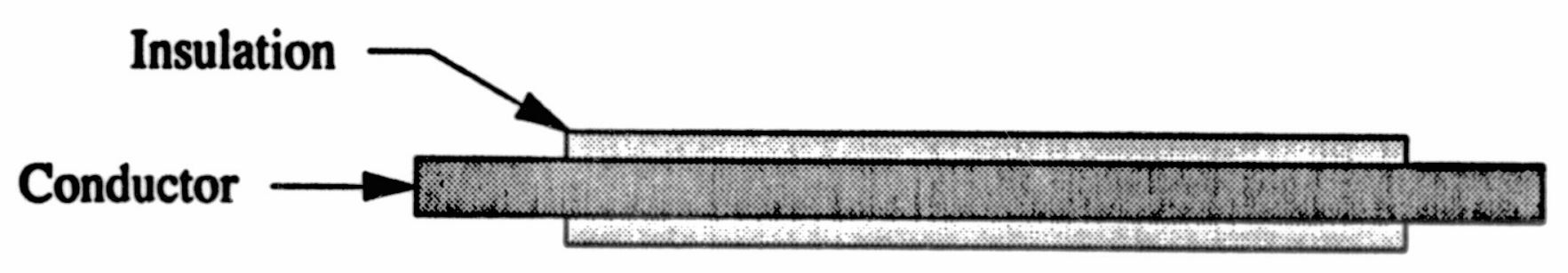

Combustion Products
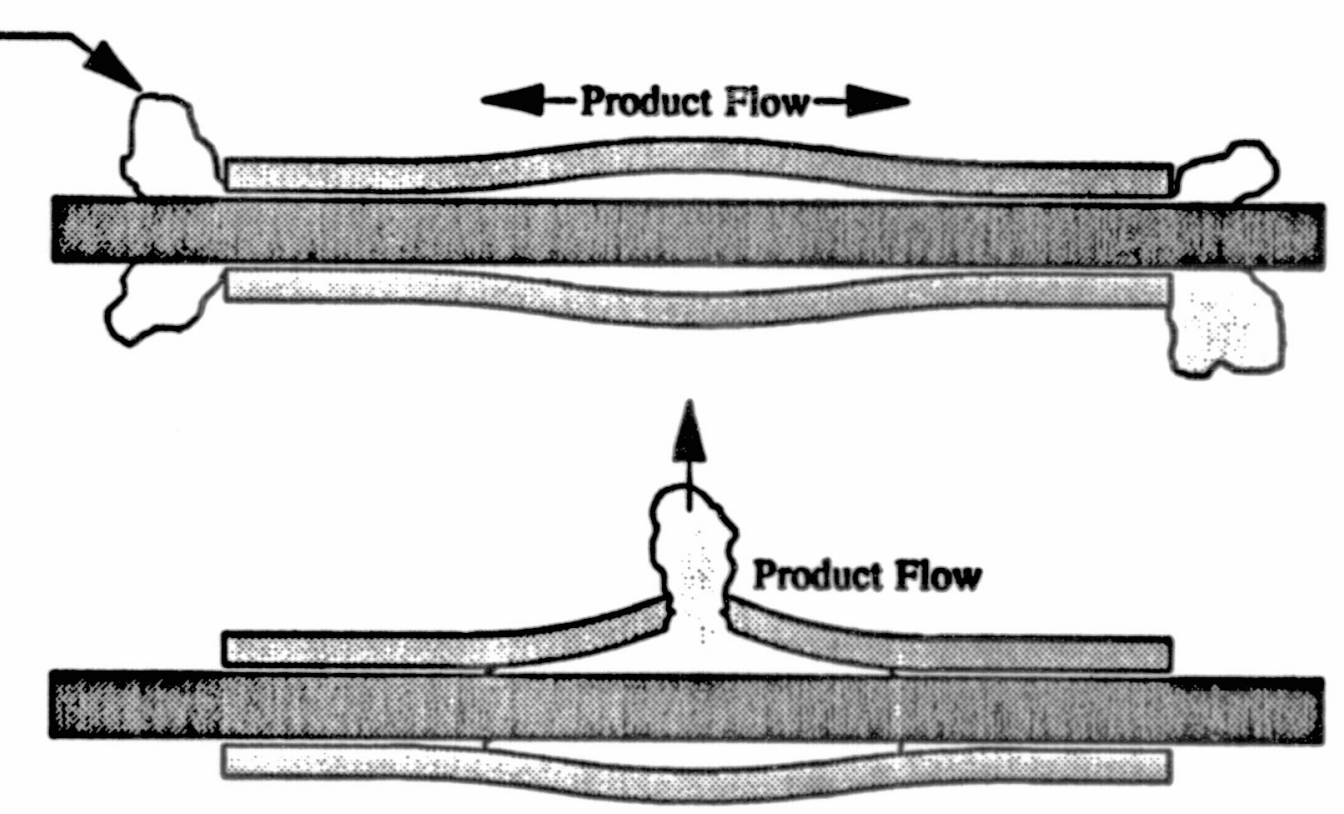


\begin{tabular}{|c|c|c|c|c|}
\hline & Damage Modes & Tests & Models & ल्लिय \\
\hline & Heat Release & temperature measurements & $f(T)$ & \\
\hline Source & Smoke Release & obscuration, TEM grids & $f(T)$ & \\
\hline & Toxin Release & IR/Mass spec.(White Sands), sampling & $f(T)$ & $:$ \\
\hline & Heat Transport - & temperature measurements & \multirow{3}{*}{\multicolumn{2}{|c|}{$\begin{array}{l}\text { fluid flow, temp., etc } \\
\text { fluid flow, temp., etc } \\
\text { fluid flow, temp., etc }\end{array}$}} \\
\hline Transport & Smoke Transport & TEM grids/visualization & & \\
\hline & Toxin Transport & & & \\
\hline & Adjacent Wire Damage & pairs, bundles & \multicolumn{2}{|c|}{ heat release, qualitative } \\
\hline Deposition & Particulate Deposition & TEM grids & \multicolumn{2}{|l|}{ TBD } \\
\hline & Cornosivity & thin copper target plate & \multicolumn{2}{|l|}{ qualliative } \\
\hline
\end{tabular}




\section{NASA Lewis $2.2 \mathrm{sec}$ Drop Tower}

Sample Materials

PTFE - Teflon

[- $\left.\mathrm{CF}_{2}-\mathrm{CF}_{2}-\right]$

ETFE - Tefzel

$\left[-\mathrm{CF}_{2}-\mathrm{CH}_{2}\right.$ ]

Bundles

Twisted Pairs
Interior wiring

Exterior wiring

Interior/Exterior

Interior/Exterior
Smoke Production

Toxic Production

Acidic Production

Combustible Production

+ Adjacent Wire Damage 


\section{PTFE \\ Polytetrafluorethylene Thermal Degradation Products}

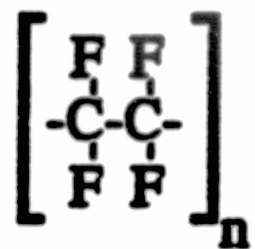

Approximate Melting Temperature

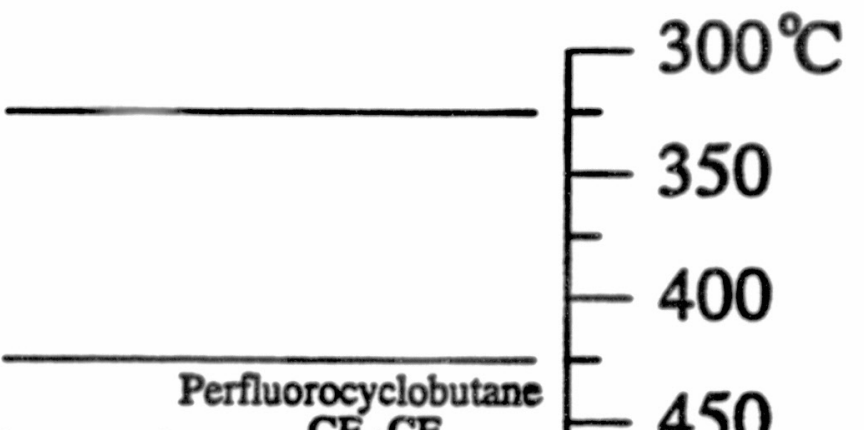

Perfluoroisobutylene

TFE Monomer

$\left(\mathrm{CF}_{3}\right)_{2} \mathrm{C}=\mathrm{CF}_{2}$

$\mathrm{CF}_{2}=\mathrm{CF}_{2}$

Hexafluorpropylene

$\mathrm{CF}_{3} \mathrm{CF}=\mathrm{CF}_{2}$

$\mathrm{CF}_{2}-\mathrm{CF}_{2}$

450

500

$\mu \mathrm{g}$

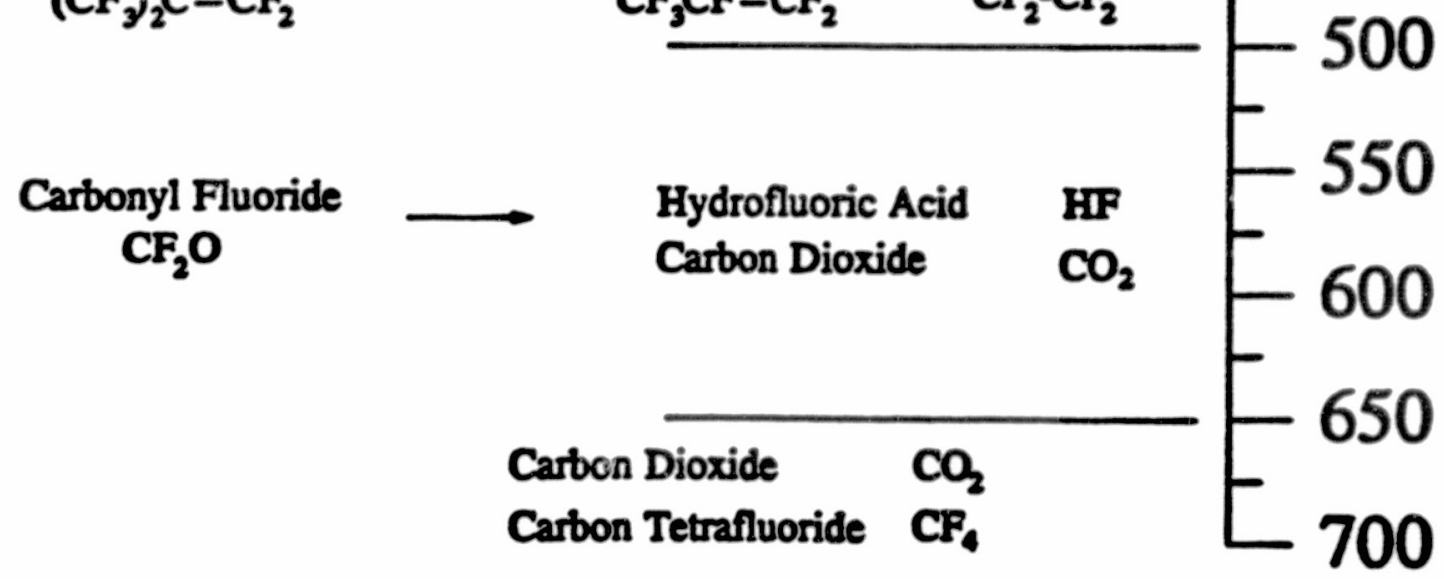

